\title{
Escoamento superficial e desagregação do solo em entressulcos em solo franco-argilo-arenoso com resíduos vegetais
}

\author{
Elemar Antonino Cassol(1), José Ramon Barros Cantalice ${ }^{(2)}$, José Miguel Reichert ${ }^{(3)}$ e Arcângelo Mondardo ${ }^{(4)}$ \\ (1)Universidade Federal do Rio Grande do Sul, Faculdade de Agronomia, Dep. de Solos, Av. Bento Gonçalves, 7712, Bairro Agronomia, Caixa \\ Postal, 15100, CEP 90001-970 Porto Alegre, RS. E-mail: cassolea@orion.ufrgs.br (2)Universidade Federal Rural de Pernambuco, Dep. de \\ Agronomia, Rua Dom Manuel Medeiros, s/no, Bairro Dois Irmãos, CEP 52171-900 Recife, PE. E-mail: cantalic@terra.com.br (3)Universidade \\ Federal de Santa Maria, Centro de Ciências Rurais, Dep. de Solos, Campus Universitário, Rua Roraima, s/no, CEP 97105-900 Santa Maria, RS. \\ E-mail: reichert@smail.ufsm.br (4)Câmara de Agronomia do CREA-RS, Rua Guilherme Alves, 1010, CEP 90680-000, Porto Alegre, RS. E-mail: \\ mondardo@terra.com.br
}

\begin{abstract}
Resumo - A presença de resíduos vegetais sobre a superfície do solo altera as características do escoamento superficial gerado pela chuva e a desagregação e transporte de sedimento resultantes do processo erosivo. O objetivo deste trabalho foi avaliar as condições hidráulicas e as relações de desagregação do solo e de resistência ao escoamento com a presença de resíduos vegetais na erosão em entressulcos. O experimento foi realizado no laboratório, com um Argissolo Vermelho distrófico típico, em parcelas com $0,10 \mathrm{~m} \mathrm{~m}^{-1}$ de declive sob chuva simulada. O solo foi coberto por resíduos vegetais de palha de soja, nas doses de $0,0,05,0,1,0,2,0,4$ e $0,8 \mathrm{~kg} \mathrm{~m}^{-2}$. O aumento na cobertura do solo (CS) com resíduos vegetais elevou a altura da lâmina de escoamento e a rugosidade hidráulica e reduziu a velocidade média do escoamento, provocada pelo aumento das forças viscosas promovida pela interposição física dos resíduos ao escoamento. O resultado é a redução na taxa de desagregação do solo $\left(D_{\mathrm{i}}\right)$. A $\mathrm{D}_{\mathrm{i}}$ foi de $5,35 \times 10^{-4} \mathrm{~kg} \mathrm{~m}^{-2} \mathrm{~s}^{-1}$ para solo descoberto e $1,50 \times 10^{-5} \mathrm{~kg} \mathrm{~m}^{-2} \mathrm{~s}^{-1} \mathrm{em}$ solo com $100 \%$ de cobertura na maior dose de palha. Os modelos de Laflen e potencial foram adequados para estimar o coeficiente de cobertura para resíduo em contato direto com a superfície do solo em função da cobertura do solo.
\end{abstract}

Termos para indexação: erosão hídrica, processos erosivos, modelagem da erosão, escoamento raso, cobertura do solo.

\section{Interrill surface runoff and soil detachment on a sandy clay loam soil with residue cover}

\begin{abstract}
Soil surface cover with crop residue modifies surface flow characteristics, generated by excess rainfall, and soil detachment and sediment transport resulting from the erosion process. The objective of this study was to evaluate the hydraulic conditions, detachment and flow resistance on interrill erosion on soil covered with residue. The experiment was conducted in the laboratory, on a Hapludult soil at a slope of $0.10 \mathrm{~m} \mathrm{~m}^{-1}$, under simulated rainfall and soil surface covered with soybean residue at the rates of $0,0.05,0.1,0.2,0.4$, and $0.8 \mathrm{~kg} \mathrm{~m}^{-2}$. The increase in soil surface cover (SC) with residue, caused an increase in water flow depth and hydraulic roughness, and a decrease in the mean flow velocity, due to an increase in the viscous forces from the physical interference of residue on runoff, thus contributing to a reduction in interrill soil detachment rate $\left(D_{i}\right)$. The $D_{i}$ was $5.35 \times 10^{-4} \mathrm{~kg} \mathrm{~m}^{-2} \mathrm{~s}^{-1}$ for bare soil and was reduced to $1.50 \times 10^{-5} \mathrm{~kg} \mathrm{~m}^{-2} \mathrm{~s}^{-1}$ for soil with $100 \%$ of surface cover. The Laflen's and the potential models were adequate to estimate the coefficient of soil coverage by residue in direct contact with soil as a function of the soil surface cover.
\end{abstract}

Index terms: water erosion, erosion processes, erosion modeling, shallow flow, mulch.

\section{Introdução}

A erosão em entressulcos é resultante do processo de desagregação das partículas do solo pelo impacto das gotas de chuva e do transporte das partículas desagregadas, pela delgada lâmina de escoamento. A capacidade de transporte do escoamento é incrementada pelo impacto das gotas de chuva (Foster, 1982).
Na erosão em entressulcos, a redução da velocidade do escoamento causada pela presença de resíduos vegetais na superfície ocorre, geralmente, por causa do aumento da rugosidade hidráulica do fluxo superficial, resultando em aumento da altura do escoamento (Foster, 1982; Braida \& Cassol, 1999).

A relação entre as taxas de desagregação e a presença de resíduos vegetais na erosão em entressulcos 
é, segundo Foster (1982):

$$
\mathrm{D}_{\mathrm{i}}=\mathrm{K}_{\mathrm{i}} \mathrm{I}^{2} \mathrm{~S}_{\mathrm{f}} \mathrm{C}_{\mathrm{i}}
$$

em que $\mathrm{D}_{\mathrm{i}}$ é a taxa de desagregação do solo $\left(\mathrm{kg} \mathrm{m}^{-2} \mathrm{~s}^{-1}\right)$; I é a intensidade da chuva $\left(\mathrm{m} \mathrm{s}^{-1}\right) ; \mathrm{S}_{\mathrm{f}}$ é o fator de declive; $\mathrm{C}_{\mathrm{i}}$ é o coeficiente de cobertura do solo.

Esse coeficiente $\mathrm{C}_{\mathrm{i}}$ combina vários subfatores, segundo a expressão:

$\mathrm{C}_{\mathrm{i}}=\mathrm{C}_{\mathrm{iI}} \mathrm{C}_{\mathrm{iII}} \mathrm{C}_{\mathrm{iIII}}$

em que $\mathrm{C}_{\mathrm{iI}}$ é o fator que expressa o efeito da cobertura da copa ou do dossel; $\mathrm{C}_{\mathrm{iII}}$ é o fator que expressa o efeito da cobertura por resíduos em contato direto com a superfície do solo; $\mathrm{C}_{\mathrm{iIII}}$ é o fator que expressa o efeito do resíduo incorporado ao solo.

O efeito de resíduos em contato direto com a superfície do solo foi avaliado por alguns autores, que propuseram relações capazes de quantificar o efeito dessa rugosidade dita de forma, que se traduz em resistência ao escoamento e, por conseguinte, em redução das taxas de desagregação de solo. Foster (1982) propôs a seguinte expressão:

$\mathrm{C}_{\mathrm{iII}}=\xi \exp \left\{-0,21\left[\left(\mathrm{y}_{\mathrm{c}} / \mathrm{y}_{\mathrm{d}}\right)-1\right]^{1,18}\right\}$

em que $\mathrm{C}_{\text {iII }}$ é o fator que expressa o efeito da cobertura por resíduos em contato direto com a superfície do solo; $\xi$ é a fração da superfície exposta ao impacto da chuva; $\mathrm{y}_{\mathrm{c}} / \mathrm{y}_{\mathrm{d}}$ é a razão entre a altura da lâmina de escoamento com cobertura $\left(\mathrm{y}_{\mathrm{c}}\right)$ e sem cobertura $\left(\mathrm{y}_{\mathrm{d}}\right)$.

Laflen et al. (1985) desenvolveram uma expressão mais simples:

$\mathrm{C}_{\mathrm{iII}}=\mathrm{e}^{-2,5 \mathrm{CS}}$

em que $\mathrm{C}_{\mathrm{iII}}$ é o fator que expressa o efeito da cobertura por resíduos em contato direto com a superfície do solo; e é a base do logaritmo neperiano; CS é a cobertura do solo $\left(\mathrm{m}^{2} \mathrm{~m}^{-2}\right)$. Braida \& Cassol (1999) observaram que essa relação se ajustou muito bem aos dados experimentais com resíduos de trigo e de milho na superfície do solo, apenas alterando o valor da constante do expoente para 2,32.

O objetivo deste trabalho foi avaliar as condições hidráulicas e as relações de desagregação do solo e de resistência ao escoamento com a presença de resíduos vegetais na erosão em entressulcos.

\section{Material e Métodos}

O experimento foi realizado no Laboratório de Erosão do Solo do Departamento de Solos da Faculdade de Agronomia da Universidade Federal do Rio Grande do
Sul (UFRGS), em parcelas experimentais com área útil de $0,3481 \mathrm{~m}^{2}$, com dimensões de $0,59 \mathrm{~m}$ por $0,59 \mathrm{~m}$ e profundidade de $10 \mathrm{~cm}$, havendo uma bordadura lateral de $20 \mathrm{~cm}$ de largura. As parcelas foram construídas com material de acrílico e montadas sobre uma estrutura metálica. O volume da parcela experimental foi preenchido com uma camada de $3 \mathrm{~cm}$ de espessura de brita, sobre a qual foi colocada uma camada de $1 \mathrm{~cm}$ de areia e, sobre essas duas, uma tela de plástico com malha de $1 \mathrm{~mm}$, sobre a qual colocou-se uma camada de solo com $6 \mathrm{~cm}$ de espessura, para uma densidade de empacotamento $\left(\mathrm{D}_{\mathrm{ep}}\right)$ de $1,0 \mathrm{Mg} \mathrm{m}^{-3}$. O solo utilizado foi um Argissolo Vermelho distrófico típico (Embrapa, 1999), passado em peneira com malha de $10 \mathrm{~mm}$ de diâmetro.

As amostras de solo nas parcelas foram saturadas com antecedência de 24 horas, mediante um conjunto de seis mangueiras acopladas no fundo da parcela e conectadas a um reservatório de água posicionando a uma altura levemente superior ao topo da parcela experimental. Ao iniciar os testes com chuva, aplicava-se uma tensão de água equivalente a $60 \mathrm{~cm}$ de coluna d'água $(60 \mathrm{cca})$, a qual era mantida ao longo do experimento. A declividade das parcelas experimentais era de $0,10 \mathrm{~m} \mathrm{~m}^{-1}$.

Resíduos de soja secados em estufa a $65^{\circ} \mathrm{C}$ foram picotados em pedaços de $1 \mathrm{~cm}$ de comprimento e distribuídos sobre a camada de solo das parcelas, nas seguintes doses: 0,0, 0,05, 0,10, 0,20, 0,40 e $0,80 \mathrm{~kg} \mathrm{~m}^{-2}$. Todas as parcelas foram fotografadas, para determinação das porcentagens de cobertura do solo, pelo método de Mannering (Lopes, 1984). As doses referidas, em três repetições, constituíram-se nos tratamentos aplicados num delineamento experimental inteiramente casualizado. Foram realizados 18 testes de chuva simulada.

As chuvas simuladas em laboratório foram efetuadas utilizando-se simulador de chuvas, semelhante ao descrito por Meyer \& Harmon (1979), com um bico aspersor tipo Veejet 80-150 com diâmetro interno de 12,7 $\mathrm{mm}$ situado a 3,1 $\mathrm{m}$ acima da superfície do solo, operando com uma pressão constante de $41 \mathrm{kPa}$ na saída de água do bico. As intensidades médias das chuvas simuladas observadas foram determinadas por um conjunto de 10 pluviômetros, colocados ao acaso ao lado da área útil das parcelas experimentais.

A velocidade superficial $\left(\mathrm{m} \mathrm{s}^{-1}\right)$ foi obtida pela tomada do tempo para um corante (azul de metileno), aplicado ao escoamento, percorrer o espaço entre o ponto de aplicação e o vertedor da parcela. Esse procedimento 
foi realizado em intervalos de 5 minutos, a partir da formação e deslocamento da lâmina de escoamento. Os valores da velocidade superficial do escoamento, assim obtidos, foram então multiplicados por um fator de correção $(\alpha=2 / 3)$, conforme Farenhorst \& Bryan (1995) e Katz et al. (1995).

A altura da lâmina do escoamento (h) foi determinada pela seguinte equação:

$\mathrm{h}=\mathrm{q} / \mathrm{V}$

em que q é a descarga líquida total por unidade de largura $\left(\mathrm{m}^{2} \mathrm{~s}^{-1}\right)$; V é a velocidade média do escoamento $\left(\mathrm{m} \mathrm{s}^{-1}\right)$; h é a altura da lâmina de escoamento (m). A descarga líquida foi determinada a partir das coletas de enxurrada em potes de plástico, colocados na extremidade inferior da calha coletora. As coletas nos potes foram efetuadas a cada dois minutos, sendo coletado todo o volume da enxurrada.

Na definição das condições hidráulicas do escoamento e de sua interação com a superfície do solo, foram determinadas características hidráulicas conforme descritas em Chow (1959) e apresentadas a seguir. O número de Reynolds foi determinado pela relação:

$\mathrm{R}_{\mathrm{e}}=\mathrm{Vh} / \mathrm{v}$

em que $\mathrm{R}_{\mathrm{e}}$ é o número de Reynolds (adimensional); $\mathrm{V}$ é a velocidade média do escoamento $\left(\mathrm{m} \mathrm{s}^{-1}\right)$; h é a altura da lâmina de escoamento $(\mathrm{m})$; $v$ é a viscosidade cinemática da água $\left(\mathrm{m}^{2} \mathrm{~s}^{-1}\right)$. A temperatura da água foi determinada em ${ }^{\circ} \mathrm{C}$ por meio de termômetro em cada ensaio e a viscosidade cinemática, em função da temperatura, foi obtida pela expressão (Julien, 1995):

$v=\left[1,14-0,031(\mathrm{~T}-15)+0,00068(\mathrm{~T}-15)^{2}\right] \times 10^{-6}$

em que $v$ é a viscosidade cinemática da água $\left(\mathrm{m}^{2} \mathrm{~s}^{-1}\right)$; $\mathrm{T}$ é a temperatura da água $\left({ }^{\circ} \mathrm{C}\right)$.

O número de Froude (Fr) foi obtido pela expressão:

$\mathrm{F}_{\mathrm{r}}=\mathrm{V} / \sqrt{\mathrm{gh}}$

em que $\mathrm{V}$ é a velocidade média do escoamento $\left(\mathrm{m} \mathrm{s}^{-1}\right)$; $\mathrm{g}$ é a aceleração da gravidade $\left(\mathrm{m} \mathrm{s}^{-2}\right)$; h é a altura da lâmina de escoamento (m). A rugosidade foi determinada pelo coeficiente de Manning (n) conforme Braida \& Cassol (1999):

$\mathrm{n}=\mathrm{h}^{5 / 3} \mathrm{~S}^{1 / 2} / \mathrm{q}$

em que né ocoeficiente de rugosidade de Manning ( $\left.\mathrm{s} \mathrm{m}^{-1 / 3}\right)$; $\mathrm{h}$ é a altura da lâmina de escoamento (m); q é a descarga líquida $\left(\mathrm{m}^{2} \mathrm{~s}^{-1}\right)$; $\mathrm{S}$ é o declive da parcela $\left(\mathrm{m} \mathrm{m}^{-1}\right)$.

As taxas de perdas de solo e a concentração de sedimentos foram obtidas pela pesagem do material coletado nos potes de plástico com capacidade de $920 \mathrm{~mL}$, colocados na extremidade inferior da calha coletora. Aos potes de plástico, após pesagem, adicionou-se $5 \mathrm{~mL}$ de alúmen de potássio a 5\%, para decantação das partículas. Após 24 horas, o sobrenadante foi sifonado e o solo foi secado em estufa a $65^{\circ} \mathrm{C}$. Em seguida os potes foram pesados com e sem o solo seco. A relação entre a massa de solo seco e a massa de mistura água-sedimento expressa a concentração de sedimentos em peso $\left(\mathrm{g} \mathrm{g}^{-1}\right)$.

As taxas de desagregação do solo em entressulcos $\left(D_{i}\right.$, em kg m$\left.{ }^{-2} \mathrm{~s}^{-1}\right)$ foram determinadas para cada intervalo de amostragem pela divisão da massa de solo desagregado na unidade de tempo, pela área da parcela $\left(0,3481 \mathrm{~m}^{2}\right)$. Como as intensidades observadas da chuva variaram em torno do valor planejado de $75 \mathrm{~mm} \mathrm{~h}^{-1}$, aplicou-se um fator de correção às taxas de desagregação do solo para normalização desses valores. $\mathrm{O}$ fator de correção consistiu, segundo Cassol et al. (1999), na seguinte expressão:

$\mathrm{D}_{\text {in }}=\mathrm{D}_{\mathrm{i}}\left(\mathrm{I}_{\mathrm{p}} / \mathrm{I}_{\mathrm{o}}\right)^{2}$

em que $\mathrm{D}_{\text {in }}$ são as taxas de desagregação do solo normalizadas $\left(\mathrm{kg} \mathrm{m}^{-2} \mathrm{~s}^{-1}\right)$; $\mathrm{D}_{\mathrm{i}}$ são as taxas de desagregação do solo observadas $\left(\mathrm{kg} \mathrm{m}^{-2} \mathrm{~s}^{-1}\right)$; $\mathrm{I}_{\mathrm{p}}$ é a intensidade de chuva planejada $\left(\mathrm{mm} \mathrm{h}^{-1}\right)$; $\mathrm{I}_{\mathrm{o}}$ é a intensidade de chuva observada $\left(\mathrm{mm} \mathrm{h}^{-1}\right)$. As perdas totais de solo em entressulcos foram obtidas pela expressão:

$\mathrm{PS}_{\mathrm{i}}=\sum \mathrm{QCt} / \mathrm{A}$

em que $\mathrm{PS}_{\mathrm{i}}$ é a perda de solo em entressulcos $\left(\mathrm{kg} \mathrm{m}^{-2}\right)$; Q é a descarga total $\left(\mathrm{kg} \mathrm{s}^{-1}\right)$; $\mathrm{C}$ é a concentração de sedimentos na enxurrada $\left(\mathrm{kg} \mathrm{L}^{-1}\right)$; té o intervalo de tempo entre duas amostragens (120 s); A é a área da parcela $\left(\mathrm{m}^{2}\right)$.

Considerando-se que, nas condições deste trabalho, não havia resíduos incorporados e nem cobertura de dossel, tem-se que $\mathrm{C}_{\mathrm{iI}}=1$ e $\mathrm{C}_{\mathrm{iIII}}=1$, de modo que a expressão (2) se reduz a $\mathrm{C}_{\mathrm{i}}=\mathrm{C}_{\mathrm{iII}}$ e o fator $\mathrm{C}_{\mathrm{iII}}$ pode ser estimado pela seguinte expressão:

$\mathrm{C}_{\mathrm{iII}}=\mathrm{D}_{\mathrm{i}} / \mathrm{K}_{\mathrm{i}} \mathrm{I}^{2} \mathrm{~S}_{\mathrm{f}}$

em que $K_{i}$ é a erodibilidade do solo em entressulcos $\left(\mathrm{kg} \mathrm{s} \mathrm{m}^{-4}\right) ; \mathrm{D}_{\mathrm{i}}$ é a taxa máxima de desagregação do solo em entressulcos $\left(\mathrm{kg} \mathrm{m}^{-2} \mathrm{~s}^{-1}\right)$; I é a intensidade da chuva $\left(\mathrm{m} \mathrm{s}^{-1}\right) ; \mathrm{S}_{\mathrm{f}}$ é o fator de declive determinado pela expressão de Elliot et al. (1989):

$\mathrm{S}_{\mathrm{f}}=1,05-0,85 \exp ^{(-4 \operatorname{sen}(\theta))}$

em que $\theta$ é o angulo do declive. $O$ fator $S_{\mathrm{f}}$ é 0,2 para declive plano e 1 para declive de $45^{\circ}$. 
$\mathrm{Na}$ análise estatística, a significância dos coeficientes de determinação das análises de regressão obtidas foi verificada pelo teste $\mathrm{F}$ a $1 \%$ de probabilidade.

\section{Resultados e Discussão}

Os regimes de escoamento em todas as doses de resíduo aplicadas foram laminar subcrítico, como indicam os valores de $\mathrm{R}_{\mathrm{e}}<500$ e $\mathrm{F}_{\mathrm{r}}<1$ (Tabela 1). Quantidades aplicadas de palha de soja superiores a $0,05 \mathrm{~kg} \mathrm{~m}^{-2}$ tiveram pouco efeito no número de Reynolds e mantiveram um valor aproximadamente constante. Contudo, com o aumento na dose de palha, os valores dos números de Froude diminuíram. Pela ação de resistência ao escoamento promovida pela rugosidade de forma, originada por conta da presença dos resíduos vegetais, há aumento das forças viscosas, que se reflete na elevação da altura da lâmina de escoamento e aumento das forças gravitacionais, que diminui o número de Froude. A redução da velocidade do escoamento e a elevação da altura de lâmina que ocorreram na presença dos resíduos vegetais estão em concordância com Foster (1982) e Braida \& Cassol (1999).

Com o incremento nas doses de palha de 0,0 a $0,8 \mathrm{~kg} \mathrm{~m}^{-2}$, os valores de rugosidade hidráulica (n), que retratam a resistência ao escoamento, elevaram-se de 0,043 a 0,522 (Tabela 1). Esses valores de rugosidade produzidos pelas altas doses de resíduo correspondem aos valores de rugosidade determinada para o escoamento em planícies de florestas inundadas com densa vegetação (Simons \& Senturk, 1992). Como a altura do escoamento nas áreas em entressulcos é da ordem de grandeza de $10^{-4} \mathrm{~m}$, as doses mais altas de resíduos produzem uma rugosidade semelhante à produzida pela densa vegetação de florestas em planícies inundadas.

A elevação da resistência ao escoamento nos respectivos aumentos das doses de palha aplicadas, retratada pelo aumento dos valores da rugosidade hidráulica (n), refletem-se na redução das taxas de desagregação $\left(D_{i}\right)$ de solo em entressulcos (Figura 1) e da perda de solo (Figura 2). A relação entre $D_{i}\left(\mathrm{~kg} \mathrm{~m}^{-2} \mathrm{~s}^{-1}\right)$ e $\mathrm{n}$ $\left(\mathrm{s} \mathrm{m}^{-1 / 3}\right)$ foi $\mathrm{D}_{\mathrm{i}}=1,197 \times 10^{-5} \mathrm{n}^{-1,185}, \mathrm{com} \mathrm{R}^{2}=0,974 * *$.

Os valores de $\mathrm{C}_{\mathrm{iII}}$ para o resíduo de soja foram determinados pela expressão (12) a partir da observação das taxas de desagregação de solo em entressulcos $\left(\mathrm{D}_{\mathrm{i}}\right)$, utilizando-se o valor de erodibilidade em entressulcos $\left(\mathrm{K}_{\mathrm{i}}\right)$ determinado por Cantalice (2001) para o solo do presente estudo de $2,55 \times 10^{6} \mathrm{~kg} \mathrm{~s} \mathrm{~m}^{-4}$ e com o fator de declive $\left(\mathrm{S}_{\mathrm{f}}\right)$ determinado pela expressão (13) de 0,4792 (Tabela 2). O incremento nas doses de resíduo de soja reduziu as taxas de desagregação do solo em entressulcos $\left(\mathrm{D}_{\mathrm{i}}\right)$ e diminuiu o valor do subfator cobertura do solo por resíduos em contato direto com a superfície do solo $\left(\mathrm{C}_{\mathrm{iII}}\right)$, tanto para o valor observado, como para os valores estimados (Tabela 2). Os modelos ajustados tiveram o mesmo coeficiente de determinação $\left(\mathrm{R}^{2}\right)$, pelo qual 99,2\% da variação no valor do fator $\mathrm{C}_{\mathrm{iII}}$ é explicada pela variação na fração de solo coberto (CS) (Tabela 3). Pela simplicidade e facilidade de aplicação, sugere-se a utilização de quaisquer dos dois modelos na estimativa do $\mathrm{C}_{\text {iII. }}$ O modelo de Laflen et al. (1985) para estimativa do efeito de resíduos culturais em contato direto com a superfície do solo, ajustado neste trabalho para resíduos da cultura de soja $\left(\mathrm{C}_{\mathrm{iII}}=\mathrm{e}^{-2,47 \mathrm{CS}}, \mathrm{R}^{2}=0,992^{* * *}\right)$, tem valores muito próximos aos obtidos por Braida \& Cassol (1999), que obtiveram $C_{\text {iII }}=\mathrm{e}^{-2,31 C S}$, com $R^{2}=0,94^{* *}$ para resíduo de trigo e $\mathrm{C}_{\mathrm{iII}}=\mathrm{e}^{-2,33 \mathrm{CS}}$, com $\mathrm{R}^{2}=0,97^{* *}$

Tabela 1. Características hidráulicas do escoamento em entressulcos perturbado pela chuva e na presença de resíduos vegetais, com declives de $0,1 \mathrm{~m} \mathrm{~m}^{-1}$. Valores médios de 3 repetições ${ }^{(1)}$.

\begin{tabular}{cccccccc}
\hline $\begin{array}{c}\text { Palha } \\
\left(\mathrm{kg} \mathrm{m}^{-2}\right)\end{array}$ & $\begin{array}{c}\text { Descarga líquida } \\
(\mathrm{q})\left(\mathrm{m}^{2} \mathrm{~s}^{-1}\right)\end{array}$ & $\begin{array}{c}\text { Velocidade } \\
\text { média }(\mathrm{V})\left(\mathrm{m} \mathrm{s}^{-1}\right)\end{array}$ & $\begin{array}{c}\text { Altura da } \\
\text { lâmina }(\mathrm{h})(\mathrm{m})\end{array}$ & Re & Fr & $\begin{array}{c}\text { Viscosidade } \\
\text { cinemática }(\mathrm{v})\left(\mathrm{m}^{2} \mathrm{~s}^{-1}\right)\end{array}$ & $\begin{array}{c}\text { Rugosidade de } \\
\text { Manning }(\mathrm{n})\left(\mathrm{s} \mathrm{m}^{-1 / 3}\right)\end{array}$ \\
\hline 0,00 & $5,84.10^{-6}$ & 0,0265 & 0,00022 & 5,03 & 0,56 & $1,16.10^{-6}$ & 0,043 \\
0,05 & $6,31.10^{-6}$ & 0,0224 & 0,00028 & 5,63 & 0,42 & $1,12.10^{-6}$ & 0,060 \\
0,10 & $4,95.10^{-6}$ & 0,0161 & 0,00031 & 2,42 & 0,29 & $2,04.10^{-6}$ & 0,089 \\
0,20 & $5,78.10^{-6}$ & 0,0105 & 0,00055 & 2,80 & 0,14 & $2,03.10^{-6}$ & 0,203 \\
0,40 & $5,75.10^{-6}$ & 0,0086 & 0,00067 & 2,37 & 0,10 & $2,42.10^{-6}$ & 0,281 \\
0,80 & $5,98.10^{-6}$ & 0,0060 & 0,00100 & 2,91 & 0,06 & $2,05.10^{-6}$ & 0,522 \\
\hline
\end{tabular}

${ }^{(1)}$ Re: número de Reynolds; Fr: número de Froude. 


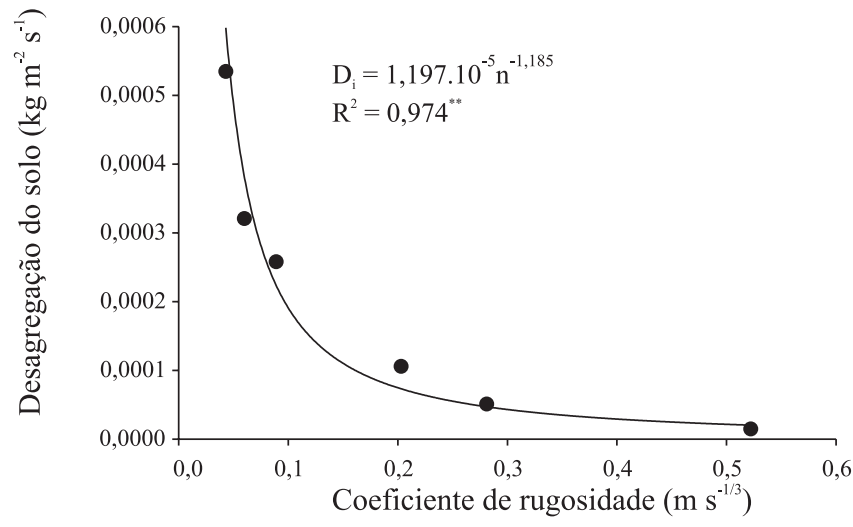

Figura 1. Taxas de desagregação do solo na erosão em entressulcos $\left(\mathrm{D}_{\mathrm{i}}\right)$, em função do coeficiente de rugosidade da superfície do solo (n) provocado pela cobertura do solo proporcionada pela palha de soja. ${ }^{* *}$ Significativo a $1 \%$ de probabilidade pelo teste $\mathrm{F}$.

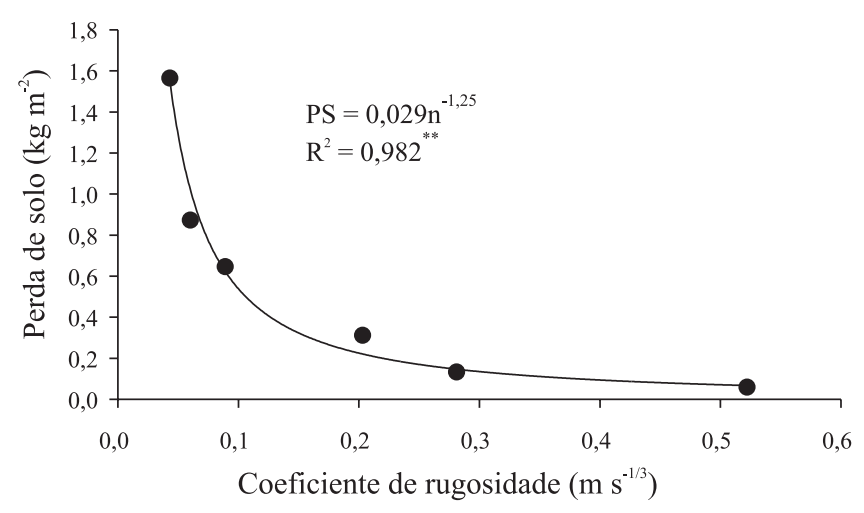

Figura 2. Perdas de solo na erosão em entressulcos (PS), em função do coeficiente de rugosidade hidráulica (n) criada pela cobertura do solo proporcionada pela palha de soja. ${ }^{* *}$ Significativo a $1 \%$ de probabilidade pelo teste $\mathrm{F}$.

Tabela 2. Taxas de desagregação $\left(\mathrm{D}_{\mathrm{i}}\right)$ ajustadas para chuva de intensidade média de $75 \mathrm{~mm} \mathrm{~h}^{-1}$ e declive de $0,10 \mathrm{~m} \mathrm{~m}^{-1}$ $\left(S_{\mathrm{f}}\right.$ de 0,4792$)$, em coberturas do solo por diferentes doses de resíduos de soja e valores observados e estimados, por duas equações, do subfator cobertura do solo em entressulcos por resíduos em contato direto com a superfície do solo $\left(\mathrm{C}_{\mathrm{iII}}\right)$.

\begin{tabular}{|c|c|c|c|c|c|}
\hline \multirow{3}{*}{$\begin{array}{c}\text { Resíduo } \\
\left(\mathrm{kg} \mathrm{m}^{-2}\right)\end{array}$} & \multirow{3}{*}{$\begin{array}{c}\text { Cobertura } \\
\left(\mathrm{m}^{2} \mathrm{~m}^{-2}\right)\end{array}$} & \multirow{3}{*}{$\begin{array}{c}\mathrm{D}_{\mathrm{i}} \\
\left(\mathrm{kg} \mathrm{m}^{-2} \mathrm{~s}^{-1}\right)\end{array}$} & \multicolumn{3}{|c|}{$\mathrm{C}_{\mathrm{iII}}$} \\
\hline & & & \multirow[t]{2}{*}{ Observado } & \multicolumn{2}{|c|}{ Estimado } \\
\hline & & & & Laflen $^{(1)}$ & Potência $^{(2)}$ \\
\hline 0,00 & 0,00 & $5,35 \cdot 10^{-4}$ & 1,00 & 1,00 & 1,00 \\
\hline 0,05 & 0,27 & $3,21.10^{-4}$ & 0,61 & 0,51 & 0,52 \\
\hline 0,10 & 0,46 & $2,58.10^{-4}$ & 0,49 & 0,32 & 0,32 \\
\hline 0,20 & 0,70 & $1,06.10^{-4}$ & 0,20 & 0,18 & 0,18 \\
\hline 0,40 & 0,92 & $5,10.10^{-5}$ & 0,10 & 0,10 & 0,10 \\
\hline 0,80 & 1,00 & $1,50 \cdot 10^{-5}$ & 0,03 & 0,08 & 0,08 \\
\hline
\end{tabular}

${ }^{(1)} \mathrm{C}_{\text {iII }}=\mathrm{e}^{-2,47 \mathrm{CS}} \cdot{ }^{(2)} \mathrm{C}_{\mathrm{iII}}=1,014 \times 0,08203^{\mathrm{CS}}$. para resíduo de milho. Esses resultados comprovam a eficiência do modelo de Laflen et al. (1985) para estimativa do efeito de resíduos culturais em contato direto com a superfície do solo na erosão em entressulcos.

A relação obtida pela análise de regressão entre valores médios de perdas de solo (PS) e a cobertura média de solo (CS) proporcionada pelas doses de palha de soja aplicadas demonstra matematicamente a redução nas perdas de solo promovida pela aplicação do resíduo de soja (Figura 3). A relação obtida entre PS $\left(\mathrm{kg} \mathrm{m}^{-2}\right)$ e CS $\left(\mathrm{m}^{2} \mathrm{~m}^{-2}\right)$ foi PS $=1,581 \mathrm{e}^{(-2,31 \mathrm{CS})}$, com $\mathrm{R}^{2}=0,984^{* *}$. Essa relação implica uma perda de solo em entressulcos aproximadamente 10 vezes maior em solo com $0 \%$ de cobertura por resíduos quando comparado a $100 \%$ de cobertura. Essa razão de aproximadamente 10 vezes também foi obtida por Cassol \& Lima (2003), que determinaram em solo descoberto ( $0 \%$ de cobertura) perdas de $2,115 \mathrm{~kg} \mathrm{~m}^{-2}$ em oposição a $0,205 \mathrm{~kg} \mathrm{~m}^{-2}$ em sistema sem preparo do solo ( $100 \%$ de cobertura).

Tabela 3. Modelos ajustados para estimativa do efeito da cobertura do solo por resíduos em contato direto com a superfície nas taxas de desagregação $\left(\mathrm{C}_{\mathrm{iII}}\right)$.

\begin{tabular}{lccc}
\hline Modelo & $\mathrm{a}$ & $\mathrm{b}$ & $\mathrm{R}^{2}$ \\
\hline Laflen: $\mathrm{C}_{\mathrm{iII}}=\mathrm{e}^{(-\mathrm{a} . \mathrm{CS})(1)}$ & 2,471 & - & $0,992 * *$ \\
Potência: $\mathrm{C}_{\mathrm{iII}}=\mathrm{ab}^{\mathrm{CS}}$ & 1,014 & 0,08203 & $0,992 * *$ \\
\hline
\end{tabular}

(1)e: base do logaritmo natural; CS: fração de solo coberto com resíduos vegetais. ${ }^{* *}$ Significativo a $1 \%$ de probabilidade pelo teste $\mathrm{F}$.

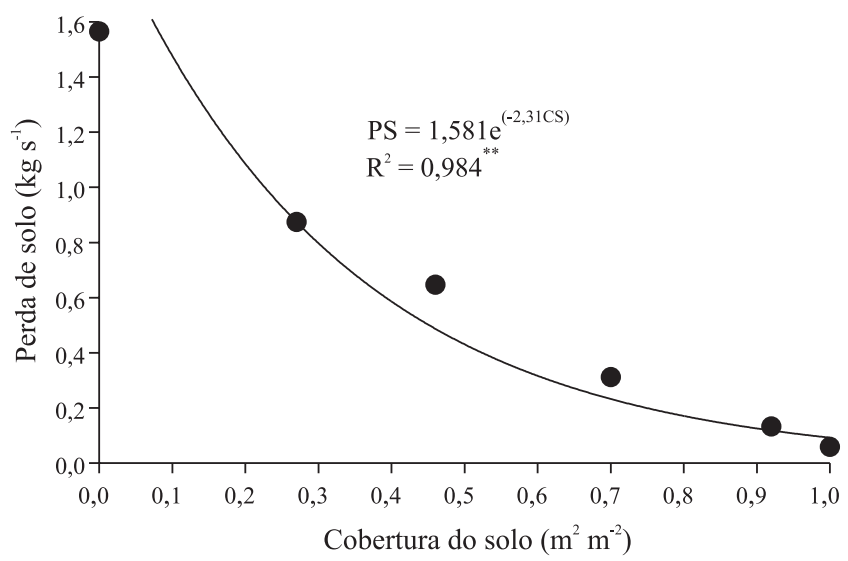

Figura 3. Perdas de solo na erosão em entressulcos (PS), em função da cobertura do solo (CS) proporcionada pela palha de soja. ${ }^{* *}$ Significativo a $1 \%$ de probabilidade pelo teste F. 


\section{Conclusões}

1. A hidráulica do escoamento em entressulcos é afetada pela presença de resíduos vegetais na superfície do solo, causando redução da velocidade e aumento da resistência e da altura da lâmina do escoamento.

2. A interposição física dos resíduos ao escoamento reduz as taxas de desagregação do solo e aumenta a resistência devido ao aumento das forças viscosas e gravitacionais, o que provoca diminuição do número de Reynolds e do número de Froude do escoamento.

3. O efeito de resíduos em contato direto com a superfície do solo sobre as taxas de desagregação em entressulcos pode ser ajustado por um modelo simples, que utiliza como único parâmetro a cobertura do solo.

\section{Agradecimentos}

Ao CNPq pela concessão de Bolsa de Pesquisa aos autores Elemar Antonino Cassol e José Miguel Reichert; ao PICDT da Capes pela concessão de Bolsa ao autor José Ramon Barros Cantalice para realização de Doutorado no PPGCS-UFRGS; à Finep e ao CNPq pelo suporte financeiro ao Projeto Pronex-Solos da UFRGS (46.61.00/2001 9); à Fapergs pelo suporte financeiro ao Projeto Temático de Erosão do Solo (Proc. № 97/0245.8); ao Técnico Agrícola Agostinho Tarcísio S. Oliveira, pela ajuda na instalação e condução dos experimentos; à Estação Experimental Agronômica da UFRGS, pela cessão da área experimental e disponibilização da infra-estrutura necessária aos trabalhos de campo.

\section{Referências}

BRAIDA, J.A.; CASSOL, E.A. Relações de erosão em entressulcos com o tipo e a quantidade de resíduo vegetal na superfície do solo. Revista Brasileira de Ciência do Solo, v.23, p.711-721, 1999.

CANTALICE, J.R.B. Escoamento e erosão em sulcos e em entressulcos em distintas condições de superfície do solo. 2001. 141p. Tese (Doutorado) - Universidade Federal do Rio Grande do Sul, Porto Alegre.

CASSOL, E.A.; LEVIEN, R.; VAN LIER, Q.J.; BADELUCCI, M.P. Infiltração de água e perdas de água e solo por erosão influenciadas por diferentes métodos de melhoramento da pastagem nativa gaúcha. Revista Brasileira de Ciência do Solo, v.23, p.923-931, 1999.

CASSOL, E.A.; LIMA, V.S. Erosão em entressulcos sob diferentes tipos de preparo e manejo do solo. Pesquisa Agropecuária Brasileira, v.38, p.117-124, 2003.

CHOW, V.T. Open-channel hydraulics. New York: McGraw-Hill, 1959. 680p.

ELLIOT, W.J.; LIEBNOW, A.M.; LAFLEN, J.M.; KOHL, K.D. A compendium of soil erodibility data from WEPP cropland soil field erodibility experiments 1987 \& 88. West Lafayette: Ohio State University; United States Department of Agriculture, 1989. (National Soil Erosion Research Laboratory Report, 3).

EMBRAPA. Centro Nacional de Pesquisa de Solos (Rio de Janeiro, RJ). Sistema brasileiro de classificação de solos. Brasília: Embrapa-SPI; Embrapa-CNPS, 1999. 412p.

FARENHORST, A.; BRYAN, R.B. Particle size distribution of sediment transported by shallow flow. Catena, v.25, p.47-62, 1995.

FOSTER, G.R. Modeling the erosion process. In: HAAN, C.T.; JOHNSON, H.P.; BRAKENSIEK, D.L. (Ed.). Hydrologic modeling of small watersheds. St. Joseph: American Society of Agricultural Engineering, 1982. p.297-380. (Monograph, 5).

JULIEN, P.Y. Erosion and sedimentation. Melbourne: Cambridge University Press, 1995. 280p.

KATZ, D.M.; WATTS, F.J.; BURROUGHS, E.R. Effects of surface roughness and rainfall impact on overland flow. Journal of Hydraulics of American Society of Civil Engineering, v.121, p.547-553, 1995.

LAFLEN, J.M.; FOSTER, G.R.; ONSTAD, C. Simulation of individual storms soil losses for modeling the impact of soil erosion on cropland productivity. In: EL-SWAFY, S.A.; MOLDENHAUER, W.C. (Ed.). Soil erosion and conservation. Ankeny: Soil Conservation Society of America, 1985. p.285-295.

LOPES, P.R.C. Relações de erosão com tipos e quantidades de resíduos culturais espalhados uniformemente sobre o solo. 1984. 119p. Dissertação (Mestrado) - Universidade Federal do Rio Grande do Sul, Porto Alegre.

MEYER, L.D.; HARMON, W.C. Multiple intensity rainfall simulator for erosion research on row sideslopes. Transactions of the American Society of Agricultural Engineering, v.22, p.100$103,1979$.

SIMONS, D.B.; SENTURK, F. Sediment transport technology: water and sediment dynamics. Littleton: Water Resources Publications, 1992. 897p

Recebido em 8 de outubro de 2003 e aprovado em 2 de abril de 2004 